\title{
Fucoidan elevates surface organic cation transporter 2 expression via upregulation of protein kinase $A$ in uric acid nephropathy
}

\author{
XINLIN WU ${ }^{1 *}$, MIANSHENG YAN ${ }^{2 *}$, TAOLI LIU ${ }^{3}$, JIANTANG LIAO $^{4}$, JIANQING ZHANG $^{2}$, \\ SHUQING CHEN ${ }^{1}$, WEI DENG ${ }^{1}$, SHIJUN ZHANG ${ }^{1}$, BAOGUO SUN ${ }^{1}$, HOUMING ZHOU $^{1}$ and BIN KE \\ Departments of ${ }^{1}$ Traditional Chinese Medicine and ${ }^{2}$ Laboratory Medicine, \\ The First Affiliated Hospital of Sun Yat-sen University, Guangzhou, Guangdong 510080; ${ }^{3}$ Department of \\ Traditional Chinese Medicine, The Fifth Affiliated Hospital of Sun Yat-sen University, Zhuhai, Guangdong 519000; \\ ${ }^{4}$ Community Health Service Center of Dongshan, Guangzhou, Guangdong 510030, P.R. China
}

Received May 3, 2016; Accepted March 31, 2017

DOI: $10.3892 /$ etm.2017.5077

\begin{abstract}
Uric acid nephropathy (UAN) is caused by excessive uric acid, and is a key risk factor for uric acid nephrolithiasis, gouty arthritis, renal diseases and cardiovascular diseases. The present study aimed to evaluate the protective effect of fucoidan, a sulfated polysaccharide component of brown algae, on UAN and to elucidate the underlying molecular mechanism. A rat model of UAN was induced by adenine treatment, and rats were then randomly assigned to control, model or fucoidan treatment groups. Hematoxylin and eosin staining of the kidney tissues of rats with UAN was subjected to conventional morphological evaluation. Cellular infiltrate in the tubules, atrophic glomeruli, tubular ectasia, granuloma hyperplasia focal fibrosis and accumulated urate crystals in the tubules of UAN rat renal tissues were observed. These symptoms of kidney damage were reduced in the fucoidan treatment group. Periodic acid methenamine silver-Masson staining was performed and the results indicated that renal interstitial fibrosis was reduced among renal tissues from the fucoidan treatment group compared with the model group. Terminal deoxynucleotidyl-transferase-mediated dUTP nick end labelling staining revealed a lower proportion of apoptotic nuclei in the kidneys of the fucoidan treatment group compared with the model group. Protein kinase A (PKA) $2 \beta$
\end{abstract}

Correspondence to: Professor Xinlin $\mathrm{Wu}$ or $\mathrm{Dr}$ Bin Ke, Department of Traditional Chinese Medicine, The First Affiliated Hospital of Sun Yat-sen University, 58 Second Zhongshan Road, Guangzhou, Guangdong 510080, P.R. China

E-mail:wuxinlin670522@yeah.net

E-mail: 18026225827@163.com

*Contributed equally

Key words: fucoidan, uric acid nephropathy, kidney damage, protein kinase A, organic cation transporter 2 and phosphorylated PKA $2 \beta$ protein levels were significantly elevated in renal tissues of the fucoidan treatment group compared with the model group $(\mathrm{P}<0.05$ and $\mathrm{P}<0.01$, respectively), suggesting that PKA expression was upregulated by fucoidan. Immunohistochemistry staining of PKA in rat renal tissues demonstrated increased expression of PKA. The surface organic cation transporter 2 (OCT2) level was significantly increased by fucoidan treatment compared with the model group $(\mathrm{P}<0.01)$, with no significant change in total OCT2 level. COS-7 cells ectopically expressing OCT2 were established. It was indicated that fucoidan was able to activate PKA and upregulate surface OCT2 in OCT2-expressing COS-7 cells. This further demonstrated that upregulation of surface OCT2 expression in OCT2-expressing cells was induced by PKA upregulation. In conclusion, fucoidan upregulated surface OCT2 expression in renal tissues to alleviate the symptoms of UAN via upregulated expression of PKA.

\section{Introduction}

Fucoidan is a sulfated polysaccharide extracted from marine brown algae and some echinoderms that has various beneficial health effects (1-3). There is a growing interest among producers and consumers in using new functional ingredients, such as fucoidan, in the diet (1). Fucoidan has been extensively studied due to it having multiple biological activities and potential applications, including anticancer, antiviral, anti-metastasis, anti-lymphangiogenesis, immune modulation, blood anticoagulant, protection from radiation damage, tissue engineering and pathogen inhibition effects (2-6).

Fucoidan may also have beneficial effects on kidney function. Previous studies have reported that fucoidan may reduce the metabolic abnormalities of diabetic rats and delay the progression of diabetic renal complications in a rat model of streptozoin-induced diabetes (7). Fucoidan has also been demonstrated to have a renoprotective role in oxalate-mediated peroxidative injury (8). Furthermore, previous studies have reported that fucoidan may alleviate the symptoms of 
chronic kidney disease in a model of subtotal nephrectomy chronic kidney disease and have a renoprotective effect on active Heymann nephritis $(9,10)$.

Although fucoidan has a protective effect against uric acid nephropathy (UAN) (11), little is known about the underlying molecular mechanism of this. In the present study, a rat model of UAN was established using adenine in order to further investigate the renoprotective role of fucoidan and its molecular mechanism. Using hematoxylin and eosin (H\&E) and periodic acid-methenamine silver (PAM)-Masson staining, it was indicated that fucoidan was able to alleviate symptoms of UAN in a rat model.

Organic cation transporter 2 (OCT2) is expressed predominantly in the kidney, at the basolateral membrane of S2 and S3 segments of the proximal tubule $(12,13)$. OCT2 is a major transporter of various organic cations from the blood stream into renal epithelial cells and is important for the cellular uptake of cationic drugs across the basolateral membrane of proximal tubule cells, including metformin, lamivudine and cimetidine (14). OCT2 is regulated by protein kinase A (PKA), phosphoionositide 3-kinase, p56lck tyrosine kinase and mitogen-activated protein kinase kinase 1 and 2 (15). To gain insight into the possible roles of PKA and OCT2 in the protective effect of fucoidan, PKA $2 \beta$, phosphorylated (p)-PKA $2 \beta$, surface OCT2 and total OCT2 protein levels were evaluated in renal tissues of a rat model of UAN in the present study. The results indicated that PKA $2 \beta$ and p-PKA $2 \beta$ levels were significantly elevated in renal tissues of the fucoidan treatment group compared with the UAN model group, suggesting that PKA was activated in the kidney tissue of fucoidan treatment group rats. Notably, the surface OCT2 level was markedly increased by fucoidan treatment with no change in total OCT2 level.

To further ascertain the role of fucoidan in regulating PKA and surface OCT2 expression, COS-7 cells ectopically expressing OCT 2 were established. The level of PKA $2 \beta$ and p-PKA $2 \beta$ and surface OCT2 was significantly increased by treatment with fucoidan compared with the control in OCT2-expressing COS-7 cells. These observations indicated that fucoidan may activate PKA and upregulate surface OCT2 in OCT2-expressing COS-7 cells. Furthermore, it was confirmed that activation of PKA with 8-bromoadenosine 3',5'-cyclic monophosphate (8-bromo-cAMP) upregulated surface OCT2 in OCT2-expressing COS-7 cells. In summary, fucoidan upregulated surface OCT2 via upregulating the expression of PKA in order to alleviate the symptoms of UAN.

\section{Materials and methods}

Rat model of UAN and experimental procedures. A total of 30 male Sprague Dawley rats (weight, 167.2 $\pm 1.9 \mathrm{~g}$; age, 6-8 weeks), were randomly assigned into three experimental groups. The rats were housed in temperature- $\left(22-25^{\circ} \mathrm{C}\right)$ and humidity-(40-70\%) controlled conditions with a 12-h light/dark cycle and ad libitum access to food and water. All rats were treated by intragastric administration. Group 2 and 3 rats were administered with $30 \mathrm{mg} / \mathrm{kg}$ adenine once a day for 18 days to establish a rat model of UAN. Group 1 was allocated as the normal control group and rats werefed an equivalent amount of carboxymethylcellulose sodium (CMC-Na; Sigma-Aldrich; Merck KGaA, Darmstadt, Germany). Rats in Group 2 were then fed with an equivalent amount of saline buffer $(30 \mathrm{mg} / \mathrm{kg}$ body weight) for 18 days to establish the disease model. Rats in Group 3 were fed with a single dose of fucoidan polysaccharide sulfate ( $400 \mathrm{mg} / \mathrm{kg}$ body weight) to establish the fucoidan treatment group.

Rats were obtained from the Experimental Animal Center of Sun Yat-sen University (Guangzhou, China). The research was carried out in accordance with the Guidelines for Human Treatment of Animals set by the Association of Laboratory Animal Sciences and the Center for Laboratory Animal Sciences at Sun Yat-sen University. The study was approved by the Committee of Biomedical Ethics of Sun Yat-sen University [SYXK (yue): 2007-0081].

Chemicals and reagents. Fucoidan polysaccharide sulfate was diluted with $10 \%$ dimethyl sulfoxide in ethanol to a final concentration of $50 \mathrm{mg} / \mathrm{ml}$. Fucoidan was purchased from South Product Co., Ltd. (Uruma, Japan). Adenine tablets were purchased from Amresco, LLC (Solon, OH, USA) and diluted with $0.15 \%$ CMC-Nato a final concentration of 3\%.Cell Surface Protein Isolation kit (K295) was purchased from BioVision, Inc., (Milpitas, CA, USA). Sulfo-NHS-SS-biotin and Avidin Agarose beads were purchased from Thermo Fisher Scientific, Inc. (Waltham, MA, USA). COS-7 cells were obtained from the Cell Bank of the Chinese Academy of Sciences (Shanghai, China) and were cultured in Dulbecco's modified Eagle's medium supplemented with $10 \%$ fetal bovine serum (both Gibco; Thermo Fisher Scientific, Inc.) and $100 \mathrm{U} / \mathrm{ml}$ penicillin and $100 \mathrm{U} / \mathrm{ml}$ streptomycin (Amresco, LLC). The cells were cultured in a $5 \% \mathrm{CO}_{2}$ atmosphere at $37^{\circ} \mathrm{C}$. 8-bromo-cAMP (Sigma-Aldrich; Merck KGaA) was used asaPKA activator. OCT2-expressing COS-7 cells were treated with PKA activator 8-bromo-cAMP $(1 \mu \mathrm{mol} / \mathrm{l})$ or phosphate-buffered saline (PBS) for $12 \mathrm{~h}$ at $37^{\circ} \mathrm{C}$.

Tissue surface protein extraction/cell surface biotinylation and western blot analysis. After treatment for 18 days, rats were euthanized by $\mathrm{CO}_{2}$ inhalation, and the renal tissues from rats in different groups were frozen and stored at $-20^{\circ} \mathrm{C}$ immediately after dissection. Renal tissues were mechanically dissociated and homogenized according to the manufacturer's instructions for the surface protein extraction kit. For COS-7 cell surface biotinylation, after treatment with fucoidan $(500 \mu \mathrm{g} / \mathrm{ml})$ or control salinefor $24 \mathrm{~h}$, cells were washed and incubated with PBS supplemented with $0.5 \mathrm{mg} / \mathrm{ml}$ sulfo-NHS-SS biotin for $40 \mathrm{~min}$ at $4^{\circ} \mathrm{C}$, and excess biotin was quenched with $50 \mathrm{mM}$ Tris-PBS buffer for $20 \mathrm{~min}$ at $4^{\circ} \mathrm{C}$. Subsequently, cells were collected, lysed in radioimmunoprecipitation buffer from BioVision, Inc., and subjected to streptavidin-agarose beads at $4^{\circ} \mathrm{C}$ for a further $3 \mathrm{~h}$. The protein samples $(30 \mu \mathrm{g})$ obtained were boiled, subjected to $12 \%$ SDS-PAGE and transferred to polyvinylidene difluoride membranes (EMD Millipore, Billerica, MA, USA). The membranes were blocked with Tris-buffered saline-Tween-20 containing 2\% bovine serum albumin (Sigma-Aldrich; Merck $\mathrm{KGaA}$ ) at room temperature for $2 \mathrm{~h}$, and the membranes were then incubated with specific antibodies against OCT2 (sc-233; Santa Cruz Biotechnology, Inc., Dallas, TX, USA; 1:1,500), PKA $2 \beta$ (sc-365615; Santa Cruz Biotechnology, Inc; 1:1,500), p-PKA $2 \beta$ (sc-293036; Santa Cruz Biotechnology, Inc.; 
1:1,000), GAPDH (ab8245; Abcam, Cambridge, UK; 1:8,000). Horseradish peroxidase-conjugated goat anti-rabbit secondary antibodies (20162; ProMab Biotechnologies, Inc., Richmond, CA, USA; 1:1,000) were used. For incubation, all antibodies were incubated at $4^{\circ} \mathrm{C}$ for $12 \mathrm{~h}$. The membranes were washed inTBST (Sigma-Aldrich; Merck KGaA) at room temperature four times between the incubations with the primary and secondary antibodies. Protein bands were detected using an enhanced chemiluminescence reaction (Biological Industries, Kibbutz Beit Haemek, Israel). The intensity of each band was quantified using Quantity One software v4.62 (Bio-Rad Laboratories, Inc., Hercules, CA, USA). All experiments were repeated three times.

$H \& E$ staining of renal tissues. Renal tissues of rats were removed, fixed with $4 \%$ paraformaldehyde in PBS at room temperature for $2 \mathrm{~h}$, decalcified for 10 days at room temperature with EDTA and embedded in paraffin for histological analysis. Kidney sections ( $5 \mu \mathrm{m}$ thick) were stained with hematoxylin for $2 \mathrm{~min}$, then eosin for $30 \mathrm{sec}$. Images of the kidney from multiple sections in each rat were observed under a light microscope with identical parameters (magnification, resolution and light intensity).

PAM-Masson staining. In brief, rat kidneys were harvested, cut along the short axis at the maximum area of the whole kidney, fixed in Carnoy's solution (Beyotime Institute of Biotechnology, Haimen, China) at $37^{\circ} \mathrm{C}$ for $2 \mathrm{~h}$, embedded in paraffin, sectioned (1 $\mu \mathrm{m}$ thick), and stained with PAM. Images of the kidney from multiple sections in each rat were observed under a light microscope with identical parameters (magnification, resolution and light intensity).

Immunohistochemistryassay for PKA. Immunohistochemistry was performed by National Engineering Center for Biochip at Shanghai (China), as described previously $(16,17)$. Immunohistochemistry was carried out with antibodies specific for PKA using anti-rat PKA (sc-365615; Santa Cruz Biotechnology, Inc.; 1:300) in blocking buffer and incubated overnight at $4^{\circ} \mathrm{C}$. Negative control sections were incubated with PBS instead of primary antibodies.

OCT2-expressing plasmid construction and transfection. Green fluorescent protein (GFP)-OCT2 and GFP-C (control) vector plasmids were purchased from OriGene Technologies, Inc., (Rockville, MD, USA) and transfected into COS-7 cells using a Lipofectamine ${ }^{\circledR} 2000$ (Invitrogen; Thermo Fisher Scientific, Inc.) reagent, according to the manufacturer's protocol.

Terminal deoxynucleotidyl-transferase-mediated dUTP nick end labelling (TUNEL) assay. Frozen renal tissue sections ( $3 \mu \mathrm{m}$ thick) were cut and mounted on 3-aminopropyltriethoxysilane-treated glass slides. The TUNEL protocol was performed using the In Situ Cell Death Detection kit (Sigma-Aldrich; Merck KGaA), according to the manufacturer's instructions. Sections were dried overnight, fixed in $1 \%$ formal dehydeat room temperature for $2 \mathrm{~h}$, washed with PBS four times for $5 \mathrm{~min}$ each, permeabilized with $0.2 \%$ Triton $\mathrm{X}-100$ at $4^{\circ} \mathrm{C}$ for $10 \mathrm{~min}$, and re-washed with PBS before
A

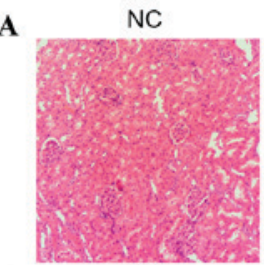

B

NC

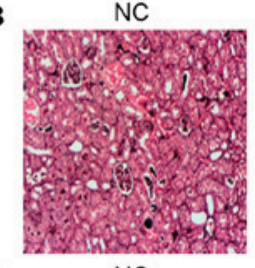

C

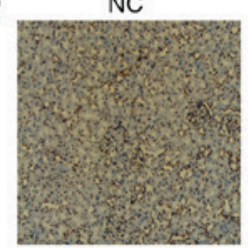

Fucoidan

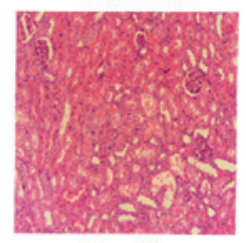

Fucoidan

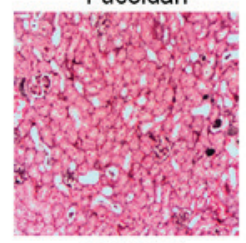

Fucoidan

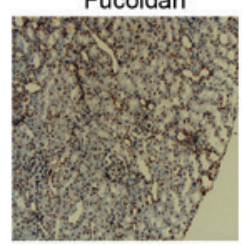

Figure 1. Fucoidan alleviates symptoms of UAN. (A) Hematoxylin and eosin staining of renal tissue from NC rats and rats with UAN. Magnification, x400. (B) Periodic acid methenamine silver-Masson staining of renal tissue from NC rats and rats with UAN. Magnification, x400. (C) Apoptotic cells in renal tissues from $\mathrm{NC}$ rats and rats with UAN, visualized using a TUNEL assay. NC, normal control; UAN, uric acid nephropathy. Magnification, x200.

application of TUNEL reagents. Images were observed under a light microscope with identical parameters (magnification, resolution and light intensity).

Statistical analysis. All data were presented as the mean \pm standard error of the mean of at least three independent experiments. Student's t-test was used to evaluate differences between two groups with GraphPad Prism v5.01 (GraphPad Software, Inc., La Jolla, CA, USA). P $<0.05$ was considered to indicate a statistically significant result.

\section{Results}

Fucoidan alleviates the symptoms of UAN in a rat model of UAN. An experimental model of UAN was induced using uricopoiesis promoter adenine. Histopathological analysis of renal tissues in each group was performed. H\&E staining of kidney tissue indicated that the control group maintained normal glomerular size, integrity and demonstrated no notable inflammatory response (Fig. 1A). In the UAN model group, cellular infiltrate in the tubules, atrophic glomeruli, tubular ectasia, granuloma hyperplasia focal fibrosis and accumulated urate crystals were observed. However, treatment with fucoidan decreased the deposition of urate crystals and inflammatory cellular influx in the tubule compared with the model group. Furthermore, tubular ectasia and interstitial fibrosis were improved by fucoidan treatment, suggesting that kidney damage was attenuated by fucoidan. The renal tissues were also processed and stained with PAM-Masson for examination under a light microscope. The PAM-Masson results revealed that renal interstitial fibrosis was less prominent among renal tissues from the fucoidan treatment group compared with the model group (Fig. 1B). In addition, the fucoidan treatment group demonstrated a lower proportion 
A

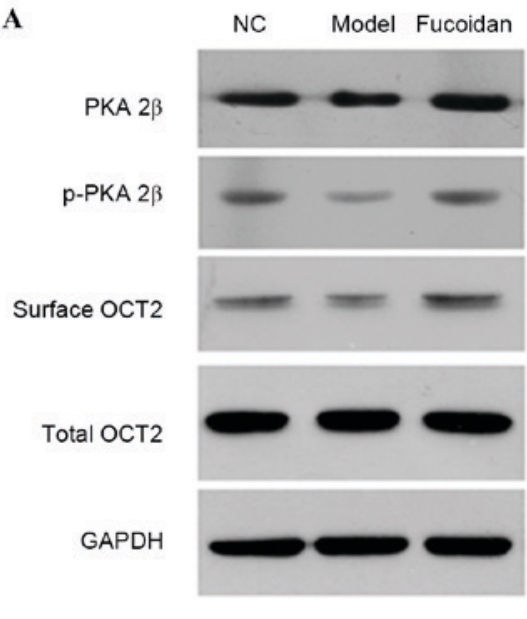

C

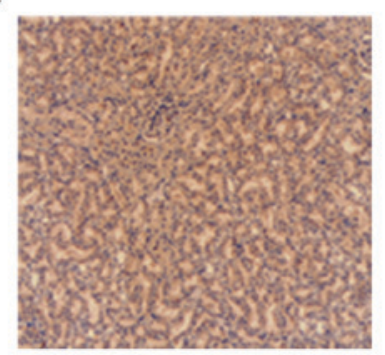

B

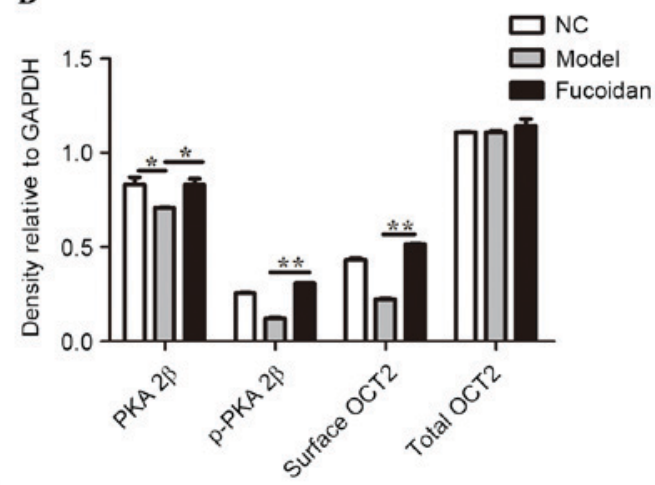

Model
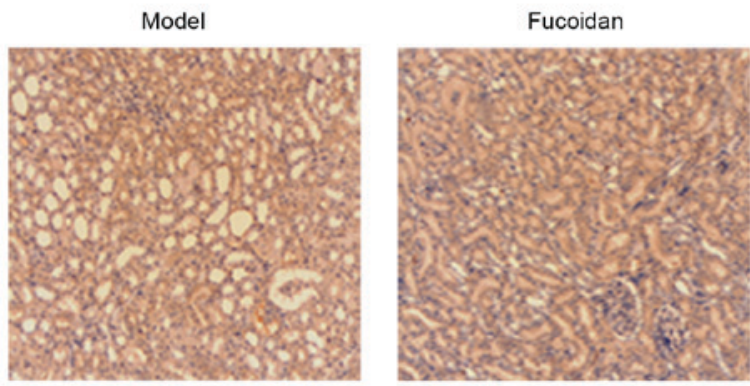

Figure 2. Fucoidan activates PKA and upregulates surface OCT2 in the renal tissue of a rat model of UAN. (A) Western blot analysis of the PKA $2 \beta$, p-PKA $2 \beta$, surface OCT2 and total OCT2 protein level in renal tissues. GAPDH was used as a loading control. (B) Densitometry plot of results from western blot analysis. Relative expression levels were normalized to GAPDH. Data are presented as the mean \pm standard error of the mean (n=3). (C) Immunohistochemistry analysis of PKA expression in kidney tissues of rats with UAN. Magnification, $\mathrm{x} 400 .{ }^{*} \mathrm{P}<0.05 ;{ }^{* *} \mathrm{P}<0.01$. PKA, protein kinase A; NC, normal control; UAN, uric acid nephropathy; OCT2, organic cation transporter 2; p, phosphorylated.

of apoptotic nuclei in the kidney compared with the model group, as assessed by TUNEL staining (Fig. 1C). These results suggested that fucoidan was able to alleviate the symptoms of UAN in a rat model.

Fucoidan activates PKA and upregulates surface OCT2 in renal tissues of a rat model of UAN. Given that fucoidan alleviated the symptoms of UAN, further study to investigate the specific molecular mechanism was required. Thus, the protein levels of PKA $2 \beta$, p-PKA $2 \beta$, surface OCT2 and total OCT 2 were evaluated in renal tissues of the different groups. As demonstrated in Fig. $2 \mathrm{~A}$ and B, PKA $2 \beta$ and p-PKA $2 \beta$ protein expression levels were significantly elevated in the renal tissues of the fucoidan treatment group compared with the model group $(\mathrm{P}<0.05$ and $\mathrm{P}<0.01$, respectively), suggesting that increased expression of PKA occurred in kidney tissue of fucoidan treatment group rats. The upregulation of PKA protein expression was further demonstrated by immunohistochemistry staining of PKA in rat renal tissues (Fig. 2C). The level of surface OCT2 protein was significantly increased by fucoidan treatment compared with the model group $(\mathrm{P}<0.01)$, with no significant change in total OCT2 level (Fig. 2A and B).

Surface OCT2 is upregulated with PKA upregulation by fucoidan in OCT2-expressing COS-7 cells. To further ascertain the role of fucoidan in regulating PKA and surface OCT2 expression, COS-7 cells ectopically expressing OCT2 were established. COS-7 cells ectopically expressing OCT2 were treated with fucoidan $(500 \mu \mathrm{g} / \mathrm{ml})$ or PBS for $24 \mathrm{~h}$. A significant increase in the proportion of surface OCT2 protein was induced by fucoidan treatment compared with the control $(\mathrm{P}<0.05$; Fig. 3$)$, with no significant difference in total OCT2 level between the two groups. A significantly elevated PKA $2 \beta$ and p-PKA $2 \beta$ level was observed in OCT2-expressing COS-7 cells treated with fucoidan compared with the control (both $\mathrm{P}<0.05$; Fig. 3). These results demonstrated that fucoidan may increase the expression of PKA and upregulate surface OCT2 in OCT2-expressing COS-7 cells.

PKA upregulates surface OCT2 in OCT-2 expressing COS-7 cells. Previous studies have reported that PKA regulates OCT2-mediated organic cation transport $(18,19)$. The increased expression of PKA by forskolin leads to stimulation of 4-[4-(dimethylamino) styryl]- $N$-methylpyridinium uptake in IHKE-1 cells (20). To study the relationship between PKA expression and surface OCT2 upregulation in COS-7 cells, surface, intracellular and total OCT2 protein levels were evaluated in OCT2-expressing COS-7 cells treated with PKA activator 8-bromo-cAMP. OCT2-expressing COS-7 cells were treated with PKA activator, 8-bromo-cAMP $(1 \mu \mathrm{mol} / \mathrm{l})$ or PBS. As demonstrated in Fig. 4, in the 8-bromo-cAMP-treated COS-7 cells, the surface OCT2 protein level was increased significantly compared with the control $(\mathrm{P}<0.05)$, while intracellular OCT2 level decreased significantly $(\mathrm{P}<0.01)$, with no significant change in total OCT2. The findings presented in the present study indicate that fucoidan may upregulate surface OCT2 via PKA to alleviate the symptoms of UAN. 

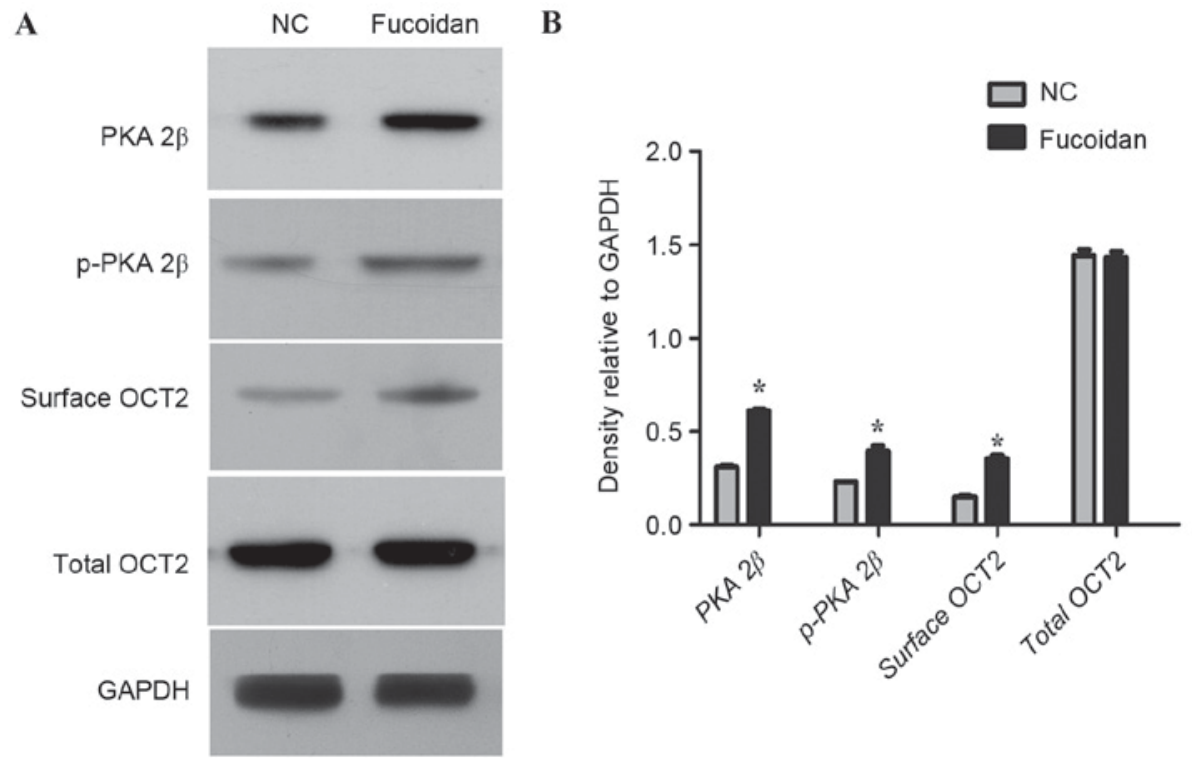

Figure 3. Surface OCT2 is upregulated by fucoidan in OCT2-expressing COS-7 cells. OCT2-expressing COS-7 cells were treated with fucoidan (500 $\mu \mathrm{g} / \mathrm{ml})$ or phosphate-buffered saline (NC) for $24 \mathrm{~h}$. (A) Western blot analysis was performed to evaluate the protein expression of PKA $2 \beta$, p-PKA $2 \beta$, total OCT2 and surface OCT2. (B) Densitometry plot of results from western blot analysis. Relative expression levels were normalized to GAPDH. Data are expressed as the mean \pm standard error of the mean $(n=3)$. ${ }^{*} \mathrm{P}<0.05$ vs. NC. PKA, protein kinase A; NC, normal control; OCT2, organic cation transporter 2; $\mathrm{p}$, phosphorylated.

A

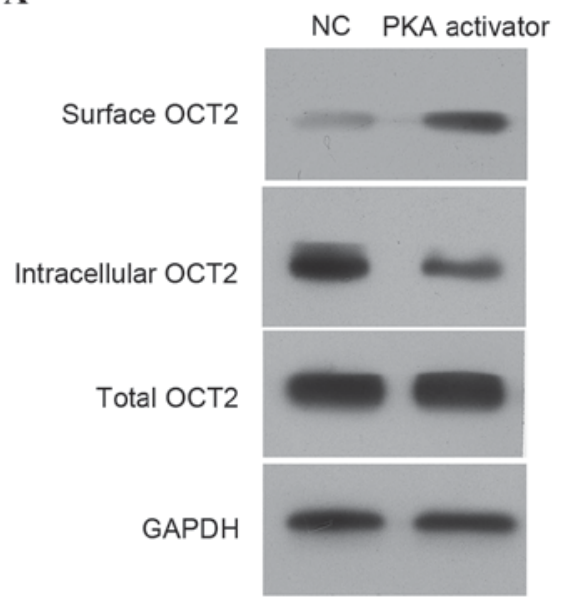

B

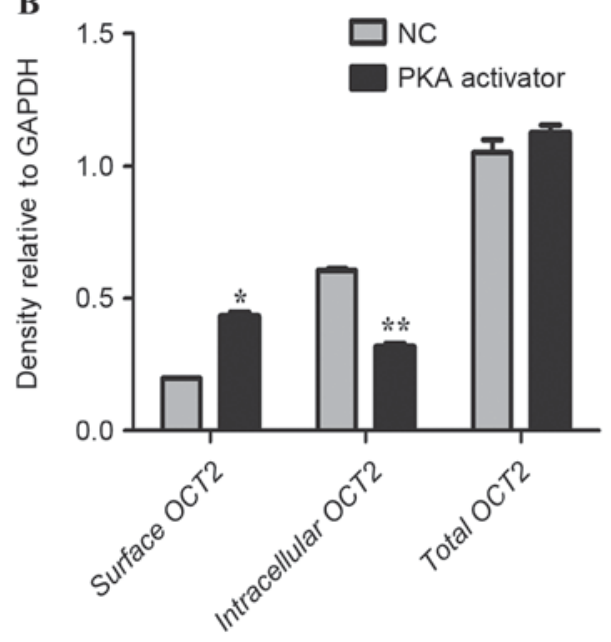

Figure 4. PKA upregulates surface OCT2 in OCT2-expressing COS-7 cells. OCT2-expressing COS-7 cells were treated with PKA activator, 8-bromoadenosine 3',5'-cyclic monophosphate (1 $\mu \mathrm{mol} / \mathrm{l})$ or phosphate-buffered saline (NC) for $12 \mathrm{~h}$. (A) Western blot analysis was performed to evaluate the protein expression of surface OCT2, total OCT2 and intracellular OCT2. (B) Densitometry plot of results from western blot analysis. Relative expression levels were normalized to GAPDH. Data are presented as the mean \pm standard error of the mean $(\mathrm{n}=3)$. ${ }^{*} \mathrm{P}<0.05$ and ${ }^{* *} \mathrm{P}<0.01 \mathrm{vs}$. NC. PKA, protein kinase A; NC, normal control; OCT2, organic cation transporter 2 .

\section{Discussion}

UAN is one of the most common and extensive metabolic diseases worldwide, and is a key risk factor for the development of uric acid nephrolithiasis, gouty arthritis, renal diseases and cardiovascular diseases, particularly hypertension (21-23). The typical characteristic of UAN is high serum uric acid level, resulting in the accumulation of urate crystals in the joints and kidneys $(24,25)$. Due to the fact that acid is the insoluble, circulating end product of purine nucleotide metabolism in humans, the urate crystals deposit in the collecting duct $(23,26)$. This induces acute kidney injury, resulting in decreased filtering capacity of the glomerulus and renal blood flow, toxic or obstructive injury to the renal tubule, edema and tubular interstitial inflammation (26).

The multiple biological activities of fucoidan have been widely studied. Its anticancer properties have been indicated by its ability to induce apoptosis and macrophage-induced tumor cell death, block the interactions between cancer cells and the basement membrane and inhibit angiogenesis by interfering with the binding of vascular endothelial growth factor and basic fibroblast growth factor $(6,27)$. The anticoagulant activity of fucoidan from Fucus evanescens was revealed in vitro and in vivo (5). In addition, fucoidan has been indicated to have a potent antiviral effect against herpes simplex virus types 1 and 2 , as well as human cytomegalovirus (28). The gastroprotective 
effect of fucoidan against aspirin-inducedulceration in rats demonstrated the immunomodulatory effect of fucoidan (29). Fucoidan also suppresses hypoxia-induced lymphangiogenesis and lymphatic metastasis in murine hepatocarcinoma by suppressing hypoxia-inducible factor-1 $\alpha /$ vascular endothelial growth factor C (30).

Consistent with previous studies, the results of the present study indicated that fucoidan was able to alleviate the symptoms of UAN in a rat model. H\&E staining revealed cellular infiltrate in the tubules, atrophic glomeruli, tubular ectasia, granuloma hyperplasia focal fibrosis and accumulated urate crystals in the tubules of renal tissues from rats with UAN, suggesting that the rat model of UAN was established successfully. The deposition of urate crystals and inflammatory cellular influx in the tubules were decreased by fucoidan administration. The proportion of kidney apoptosis was decreased by fucoidan treatment compared with the model group, as evaluated by a TUNEL staining assay. The protein levels of PKA $2 \beta$, p-PKA $2 \beta$, surface OCT2 and total OCT2 were evaluated in renal tissues. It was indicated that both the PKA $2 \beta$ and p-PKA $2 \beta$ level were significantly elevated in renal tissues of the fucoidan treatment group compared with the UAN model group. These results suggested that increased expression of PKA occurred in the kidney tissue of fucoidan treatment group rats. The upregulated protein level of PKA was further demonstrated by immunohistochemical staining of PKA in rat renal tissues. Furthermore, the surface OCT2 level was significantly increased by fucoidan treatment compared with the model group, with no significant change in total OCT2 level.

OCT2 is critical for organic cation transport, absorption and excretion of endogenous and exogenous cationic substances and urinary excretion (31-33). COS-7 cells ectopically expressing OCT2 were established in the present study to further evaluate the role of fucoidan in regulating PKA expression and surface OCT2 expression. It was indicated that fucoidan was able to increase the expression of PKA and upregulate surface OCT2 in OCT2-expressing COS-7 cells. The relationship between PKA and surface OCT2 upregulation in COS-7 cells was also investigated. The increased expression of PKA induced by 8-bromo-cAMP increased surface OCT2 and decreased intracellular OCT2 protein levels compared with the control, with no significant change in total OCT2. Consistent with previous studies, the upregulation of PKA stimulated OCT2 activity $(18,20)$.

Previous studies have reported that PKA regulates OCT2 activity in both a heterologous cell system and intact renal proximal tubules (18-20). The activation of PKA by forskolin stimulated rabbit OCT2 activity, while inhibition of PKA (by H-89) reduced transport activity of rabbit OCT2 in CHO-K1 cells (20). However, human OCT2 was reported to be inhibited by PKA activation and activated by a calmodulin-dependent signaling pathway in human embryonic kidney HEK-293 cells (34).

In conclusion, the present study identified that fucoidan upregulated surface OCT2 expression in renal tissues. This suggests that fucoidan has a protective effect against UAN may have a potential use in treating UAN. Further in-depth study of the mechanism by which fucoidan regulates human OCT2 expression and PKA activation is required.

\section{Acknowledgements}

The present study was supported by the Guangdong Provincial Department of Science and Technology (grant no. 2011B080701007).

\section{References}

1. Cunha L and Grenha A: Sulfated seaweed polysaccharides as multifunctional materials in drug delivery applications. Mar Drugs 14: pii: E42, 2016.

2. Rocha de Souza MC, Marques CT, Guerra Dore CM, Ferreira da Silva FR, Oliveira Rocha HA and Leite EL: Antioxidant activities of sulfated polysaccharides from brown and red seaweeds. J Appl Phycol 19: 153-160, 2007.

3. Fitton JH, Stringer DN and Karpiniec SS: Therapies from Fucoidan: An update. Mar Drugs 13: 5920-5946, 2015.

4. Hayashi S, Itoh A, Isoda K, Kondoh M, Kawase M and Yagi K: Fucoidan partly prevents CCl4-induced liver fibrosis. Eur J Pharmacol 580: 380-384, 2008.

5. Kuznetsova TA, Besednova NN, Mamaev AN, Momot AP, Shevchenko NM and Zvyagintseva TN: Anticoagulant activity of fucoidan from brown algae Fucus evanescens of the Okhotsk Sea. Bull Exp Biol Med 136: 471-473, 2003.

6. Koyanagi S, Tanigawa N, Nakagawa H, Soeda S and Shimeno H: Oversulfation of fucoidan enhances its anti-angiogenic and antitumor activities. Biochem Pharmacol 65: 173-179, 2003.

7. Wang J, Liu H, Li N, Zhang Q and Zhang H: The protective effect of fucoidan in rats with streptozotocin-induced diabetic nephropathy. Mar Drugs 12: 3292-3306, 2014.

8. Veena CK, Josephine A, Preetha SP, Varalakshmi P and Sundarapandiyan R: Renal peroxidative changes mediated by oxalate: The protective role of fucoidan. Life Sci 79: 1789-1795, 2006.

9. Zhang Q, Li N, Zhao T, Qi H, Xu Z and Li Z: Fucoidan inhibits the development of proteinuria in active Heymann nephritis. Phytother Res 19: 50-53, 2005.

10. Zhang Q, Li Z, Xu Z, Niu X and Zhang H: Effects of fucoidan on chronic renal failure in rats. Planta Med 69: 537-541, 2003.

11. Veena CK, Josephine A, Preetha SP and Varalakshmi P: Physico-chemical alterations of urine in experimental hyperoxaluria: A biochemical approach with fucoidan. J Pharm Pharmacol 59: 419-427, 2007.

12. Okuda M, Saito H, Urakami Y, Takano M and Inui K: cDNA cloning and functional expression of a novel rat kidney organic cation transporter, OCT2. Biochem Biophys Res Commun 224: 500-507, 1996.

13. Karbach U, Kricke J, Meyer-Wentrup F, Gorboulev V, Volk C, Loffing-Cueni D, Kaissling B, Bachmann S and Koepsell H: Localization of organic cation transporters OCT1 and OCT2 in rat kidney. Am J Physiol Renal Physiol 279: F679-F687, 2000.

14. Muller $\mathrm{F}$ and Fromm MF: Transporter-mediated drug-drug interactions. Pharmacogenomics 12: 1017-1037, 2011.

15. Wilde S, Schlatter E, Koepsell H, Edemir B, Reuter S, Pavenstadt H, Neugebauer U, Schroter R, Brast S and Ciarimboli G: Calmodulin-associated post-translational regulation of rat organic cation transporter 2 in the kidney is gender dependent. Cell Mol Life Sci 66: 1729-1740, 2009.

16. Wu YR, Qi HJ, Deng DF, Luo YY and Yang SL: MicroRNA-21 promotes cell proliferation, migration, and resistance to apoptosis through PTEN/PI3K/AKT signaling pathway in esophageal cancer. Tumour Biol 37: 12061-12070, 2016.

17. Djordjevic B, Hennessy BT, Li J, Barkoh BA, Luthra R, Mills GB and Broaddus RR: Clinical assessment of PTEN loss in endometrial carcinoma: Immunohistochemistry outperforms gene sequencing. Mod Pathol 25: 699-708, 2012.

18. Zhang Q, Li S, Patterson C and You G: Lysine 48-linked polyubiquitination of organic anion transporter-1 is essential for its protein kinase C-regulated endocytosis. Mol Pharmacol 83: 217-224, 2013.

19. Holle SK, Ciarimboli G, Edemir B, Neugebauer U, Pavenstadt H and Schlatter E: Properties and regulation of organic cation transport in freshly isolated mouse proximal tubules analyzed with a fluorescence reader-based method. Pflugers Arch 462: 359-369, 2011.

20. Soodvilai S, Chatsudthipong A and Chatsudthipong V: Role of MAPK and PKA in regulation of rbOCT2-mediated renal organic cation transport. Am J Physiol Renal Physiol 293: F21-F27, 2007. 
21. Klemp P, Stansfield SA, Castle B and Robertson MC: Gout is on the increase in New Zealand. Ann Rheum Dis 56: 22-26, 1997.

22. Arromdee E, Michet CJ, Crowson CS, O'Fallon WM and Gabriel SE: Epidemiology of gout: Is the incidence rising? J Rheumatol 29: 2403-2406, 2002.

23. Li Y, Stamler J, Xiao Z, Folsom A, Tao S and Zhang H: Serum uric acid and its correlates in Chinese adult populations, urban and rural, of Beijing. The PRC-USA collaborative study in cardiovascular and cardiopulmonary epidemiology. Int J Epidemiol 26: 288-296, 1997.

24. Johnson RJ, Kang DH, Feig D, Kivlighn S, Kanellis J, Watanabe S, Tuttle KR, Rodriguez-Iturbe B, Herrera-Acosta J and Mazzali M: Is there a pathogenetic role for uric acid in hypertension and cardiovascular and renal disease? Hypertension 41: 1183-1190, 2003.

25 . Tseng $\mathrm{CH}$ : Independent association of uric acid levels with peripheral arterial disease in Taiwanese patients with Type 2 diabetes. Diabet Med 21: 724-729, 2004.

26. Shanley PF, Rosen MD, Brezis M, Silva P, Epstein FH and Rosen S: Topography of focal proximal tubular necrosis after ischemia with reflow in the rat kidney. Am J Pathol 122: 462-468, 1986.

27. Soeda S, Kozako T, Iwata $K$ and Shimeno H: Oversulfated fucoidan inhibits the basic fibroblast growth factor-induced tube formation by human umbilical vein endothelial cells: Its possible mechanism of action. Biochim Biophys Acta 1497: 127-134, 2000.

28. Lee JB, Hayashi K, Hashimoto M, Nakano T and Hayashi T: Novel antiviral fucoidan from sporophyll of Undaria pinnatifida (Mekabu). Chem Pharm Bull (Tokyo) 52: 1091-1094, 2004.
29. RaghavendranHR, Srinivasan Pand Rekha S: Immunomodulatory activity of fucoidan against aspirin-induced gastric mucosal damage in rats. Int Immunopharmacol 11: 157-163, 2011.

30. Teng H, Yang Y, Wei H, Liu Z, Liu Z, Ma Y, Gao Z, Hou L and Zou X: Fucoidan suppresses Hypoxia-Induced lymphangiogenesis and lymphatic metastasis in mouse hepatocarcinoma. Mar Drugs 13: 3514-3530, 2015.

31. Sweet DH, Miller DS and Pritchard JB: Basolateral localization of organic cation transporter 2 in intact renal proximal tubules. Am J Physiol Renal Physiol 279: F826-F834, 2000.

32. Terashita S, Dresser MJ, Zhang L, Gray AT, Yost SC and Giacomini KM: Molecular cloning and functional expression of a rabbit renal organic cation transporter. Biochim Biophys Acta 1369: 1-6, 1998.

33. Grundemann D, Babin-Ebell J, Martel F, Ording N, Schmidt A and Schomig E: Primary structure and functional expression of the apical organic cation transporter from kidney epithelial LLC-PK1 cells. J Biol Chem 272: 10408-10413, 1997.

34. Cetinkaya I, Ciarimboli G, Yalcinkaya G, Mehrens T, Velic A, Hirsch JR, Gorboulev V, Koepsell H and Schlatter E: Regulation of human organic cation transporter hOCT2 by PKA, PI3K, and calmodulin-dependent kinases. Am J Physiol Renal Physiol 284: F293-F302, 2003. 